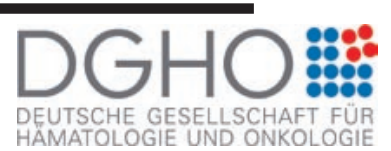

Deutsche Gesellschaft für Hämatologie und Onkologie e. V.

Geschäftsführender Vorsitzender Prof. Dr. Gerhard Ehninger

Alexanderplatz 1, 10178 Berlin, Germany

Tel. +49 302787 6089-0, Fax -18 oder -17

dgho-berlin@dgho.de

Fachgesellschaften der Onkologen begrüßen die Entscheidung des Gemeinsamen Bundesausschusses zur Stammzelltransplantation

Die Deutsche Gesellschaft für Hämatologie und Onkologie e.V. (DGHO), die Gesellschaft für Pädiatrische Onkologie und Hämatologie e.V. (GPOH) sowie die Deutsche Knochenmarkspenderdatei (DKMS, www.dkms.de) begrüßen ausdrücklich die Entscheidung des Gemeinsamen Bundesausschusses (G-BA), die Stammzelltransplantation bei Schwerer Aplastischer Anämie im Leistungskatalog der gesetzlichen Krankenkassen zu belassen.

Fünf Jahre nach der Entscheidung des G-BA, die Stammzelltransplantation bei Schwerer Aplastischer Anämie einer Nutzenbewertung durch das Institut für Qualität und Wirtschaftlichkeit im Gesundheitswesen (IQWiG) zu unterziehen, liegt seit gestern die Entscheidung vor: Die Krankenkassen werden bei einem Versagen des immunsuppressiven Therapieansatzes und fehlendem Familienspender die Transplantation von Blutstammzellen eines Fremdspenders auch zukünftig bezahlen. «Der Beschluss des Gemeinsamen Bundesausschuss bestätigt nun, was wir in den letzten Jahren immer wieder betont haben», stellt Professor Gerhard Ehninger, Vorsitzender der DGHO, fest. «Bei fehlendem Familienspender und einer fehlenden Wirksamkeit der Standardtherapie (immunsuppressiven Therapie) ist die Blutstammzell-Transplantation die einzige Heilungsmöglichkeit. Mit dieser Behandlung können wir immer noch zwei von drei Patienten von dieser schweren Erkrankung, die jeden von uns treffen kann, heilen.» Die Fachgesellschaft hatte vor der Entscheidung des G-BA in Stellungnahmen immer wieder die Wirksamkeit der allogenen Stammzelltransplantation in dieser Krankheitssituation betont und anderslautende Positionen des IQWiG kritisiert.

Auch der Vorsitzende der GPOH, Professor Thomas Klingebiel, begrüßt die Entscheidung des Gemeinsamen Bundesausschusses: «Wir freuen uns für die betroffenen Kinder,
Jugendlichen und ihre Familien, dass die Stammzelltransplantation als Therapie bei der Schweren Aplastischen Anämie auch künftig möglich ist.» - «Auch für die über vier Millionen Bundesbürger, die bereit sind, für betroffene Patienten Blutstamm- oder Knochenmarkzellen zu spenden, ist dies ein besonderer Tag», freut sich Professor Ehninger. «Durch die Diskussion in den letzten Jahren und die Infragestellung der Stammzelltransplantationen bei bestimmten Erkrankungen waren viele Spender verunsichert. Durch die Entscheidung des Gemeinsamen Bundesausschusses vom 28. Mai wird nun das Engagement der spendenwilligen Bürger und der dahinter stehenden Organisationen gewürdigt.»

\section{Weitere Informationen}

Deutsche Gesellschaft für Hämatologie und Onkologie e.V. DGHO Hauptstadtbüro

V.i.S.d.P. Mirjam Renz Fon: 030 / $27876089-0$ Fax: 030 / $27876089-18$ renz@dgho.de www.dgho.de

\section{KARGER}

Fax +497614520714

Information@Karger.de

www.karger.com

\section{(c) 2009 S. Karger GmbH, Freiburg}

Accessible online at:

www.karger.com/onk 